\title{
Distinct Gene Expression Profiles Define Anaplastic Grade in Retinoblastoma
}

Lauren E. Hudson, ${ }^{*}$ Pia Mendoza, ${ }^{* \dagger}$ William H. Hudson, ${ }^{\ddagger}$ Alison Ziesel, ${ }^{*}$ G. Baker Hubbard, III, ${ }^{*}$ Jill Wells, ${ }^{*}$ Bhakti Dwivedi, Jeanne Kowalski, ${ }^{\S \Phi}$ Sandra Seby, ${ }^{\S}$ Viren Patel, Eldon Geisert, ${ }^{*}$ Charles Specht, ** and Hans E. Grossniklaus ${ }^{* \dagger}$

From the Department of Ophthalmology, * the Winship Cancer Institute, ${ }^{\S}$ and the Department of Biostatistics and Bioinformatics, ${ }^{\circledR}$ Rollins School of Public Health, Emory University, Atlanta, Georgia; the Departments of Pathology and Laboratory Medicine ${ }^{\dagger}$ and Microbiology and Immunology, ${ }^{\ddagger}$ Emory University School of Medicine, Atlanta, Georgia; the Emory University Integrated Computational Core," Atlanta, Georgia; and the Department of Pathology, ** Penn State Milton S. Hershey Medical Center, Hershey, Pennsylvania

Accepted for publication June 13, 2018.

Address correspondence to Hans E. Grossniklaus, M.D., M.B.A., L.F. Montgomery Laboratory of Ophthalmic Pathology, Emory Eye Center, Room BT 428, 1365 Clifton Rd. NE., Atlanta,

GA 30322. E-mail: ophtheg@ emory.edu.

\begin{abstract}
Morbidity and mortality associated with retinoblastoma have decreased drastically in recent decades, in large part owing to better prediction of high-risk disease and appropriate treatment stratification. Highrisk histopathologic features and severe anaplasia both predict the need for more aggressive treatment; however, not all centers are able to assess tumor samples easily for the degree of anaplasia. Instead, identification of genetic signatures that are able to distinguish among anaplastic grades and thus predict high- versus low-risk retinoblastoma would facilitate appropriate risk stratification in a wider patient population. A better understanding of genes dysregulated in anaplasia also would yield valuable insights into pathways underlying the development of more severe retinoblastoma. Here, we present the histopathologic and gene expression analysis of 28 retinoblastoma cases using microarray analysis. Tumors of differing anaplastic grade show clear differential gene expression, with significant dysregulation of unique genes and pathways in severe anaplasia. Photoreceptor and nucleoporin expression in particular are identified as highly dysregulated in severe anaplasia and suggest particular cellular processes contributing to the development of increased retinoblastoma severity. A limited set of highly differentially expressed genes also are able to predict severe anaplasia accurately in our data set. Together, these data contribute to the understanding of the development of anaplasia and facilitate the identification of genetic markers of high-risk retinoblastoma. (Am J Pathol 2018, 188: 2328-2338; https://doi.org/10.1016/ j.ajpath.2018.06.013)
\end{abstract}

Retinoblastoma is the most common intraocular cancer of childhood, accounting for $6.1 \%$ of all cancers in children younger than 5 years of age. ${ }^{1}$ Advances in surveillance and vision-sparing techniques over the past several decades have greatly improved outcomes, ${ }^{2}$ with 5 -year survival rates now greater than $95 \%$ in developed countries. ${ }^{3}$ Depending on the level of risk and stage of retinoblastoma at presentation, eyes with retinoblastoma may be salvaged using chemoreduction, in which chemotherapy is systemically infused for five to six cycles and the primary retinoblastoma is consolidated by laser photocoagulation in the operating room. ${ }^{4}$ Alternatively, super-selective ophthalmic artery endovascular infusion of chemotherapy via intra-arterial chemotherapy is used in some centers. ${ }^{5-8}$ Both chemoreduction and intra-arterial chemotherapy have reduced enucleation rates to approximately $10 \%$ of retinoblastoma cases in developed countries. Such improvement in treatments and clinical outcomes stems from a better understanding of the risk factors associated with metastasis and mortality, which have in turn enabled better patient risk stratification and tailoring of care.

To date, estimates of clinical risk in retinoblastoma have been determined by a combination of clinical and histopathologic features. Clinical features such as older age at presentation, symptom duration longer than 6 months, poor visual acuity at presentation, buphthalmos, secondary

Supported by National Eye Institute grant NEI P30EY06360 (H.E.G.) and a grant from Research to Prevent Blindness.

Disclosures: None declared. 
glaucoma, iris neovascularization, ectropion uveae, orbital cellulitis, and group E tumors (the most severe according to the International Classification of Intraocular Retinoblastoma ${ }^{9}$ ) have been shown to be associated strongly with highrisk retinoblastoma. ${ }^{10}$ High-risk histopathologic features (HRPFs) known to be associated with worse prognosis include tumor invasion of the optic nerve, choroid, or anterior chamber and indicate the need for adjuvant chemotherapy after enucleation. ${ }^{11-13}$ However, a small percentage of retinoblastoma cases that progress to metastasis or death are not captured by these well-validated clinical and histopathologic indicators, prompting a search for additional histologic and genetic markers capable of more accurately predicting highrisk retinoblastoma and the need for chemotherapy. ${ }^{14}$

Cellular anaplasia recently was identified as an additional histopathologic indicator complementary to HRPFs in detecting high-risk retinoblastoma. ${ }^{15}$ Anaplasia, an entity similar to but distinct from cellular differentiation, is defined by specific and measurable cytologic features such as pleomorphism, hyperchromatic nuclei, and a high nucleus-to-cytoplasm ratio. ${ }^{15}$ Anaplasia is commonly used in the classification of pediatric medulloblastoma, another embryonal central nervous system tumor, which is known to be associated with aggressive clinical behavior. ${ }^{16}$ We recently determined that cellular anaplasia similarly can indicate high-risk retinoblastoma and serve as a predictor of metastases even in the absence of HRPFs. ${ }^{15}$ However, there are a limited number of ophthalmic pathology laboratories worldwide that are capable of properly examining enucleated eyes for anaplasia and HRPFs, preventing many clinical centers from taking advantage of this predictive tool.

The goal of the present study was to determine the gene expression profiles of different grades of anaplasia. Identifying differentially expressed genes may lend insight into the pathways contributing to increased anaplastic severity and also serve as the first step in defining genetic markers of anaplastic grade. Determining the gene expression profiles that distinguish anaplastic grades may lead to a gene-based clinical test to facilitate risk stratification and treatment management of retinoblastoma patients. Here, we present the gene expression profiles of 28 retinoblastoma samples of mild, moderate, and severe grade anaplasia in addition to normal retina and retinocytoma, a benign precursor of retinoblastoma. We furthermore identified genes that are differentially expressed in severe versus mild and moderate anaplasia and found that although mild and moderate anaplasia are highly similar in terms of gene expression, severe anaplasia showed significant changes in the expression of nonoverlapping genes and pathways. We describe these genetic pathways that appear to distinguish severe versus mild and moderate anaplasia and discuss how they may underlie increased severity in retinoblastoma. Finally, we determined particular genes that are able to predict severe anaplasia among our retinoblastoma samples. These data comprise the first genetic characterization of anaplasia in retinoblastoma and advance our understanding of this important predictor of severity in this childhood cancer.

\section{Materials and Methods}

\section{Samples and Patient Data}

Enucleation and exenteration specimens with a diagnosis of retinoblastoma were identified from the LF Montgomery Laboratory at the Emory Eye Center from January 1, 1940, to August 31, 2013. Exclusion criteria included any treatment before enucleation, fewer than two low-power $(20 \times)$ fields of tumor, or retinoblastoma with diffuse growth patterns. ${ }^{17,18}$ Patient medical records were reviewed for $R B 1$ mutational status (heritable or sporadic) and demographic parameters, including age at presentation, age at enucleation, sex, and race. Clinical findings including laterality, symptoms, ophthalmologic findings, Reese-Ellsworth Classification, ${ }^{19}$ International Classification of Retinoblastoma, ${ }^{20}$ length of follow-up evaluation, and treatments also were reviewed. Clinical outcomes of interest as determined from chart review were local recurrence and the presence of distant metastasis and secondary tumors. Study approval was obtained from the Emory University Institutional Review Board.

\section{Histopathologic Grading}

Histopathologic evaluation of enucleation specimens was conducted as previously described to determine tumor size, growth pattern, level of differentiation, degree of apoptosis, grade of anaplasia, tumor seeding, extent of tissue invasion, and presence of retinocytoma. ${ }^{15}$ Two ophthalmic pathologists (C.S. and H.E.G.) blinded to patient data reviewed standard pupil-optic nerve sections and transverse optic nerve sections at the surgical margin of each specimen according to American Joint Committee on Cancer pathologic classifications. ${ }^{21,22}$ Calottes were obtained and examined for all cases collected subsequent to protocol standardization [retinoblastoma (RB) 7, RB21-24, RB30, RB32, RB33]. ${ }^{21}$ Calotte

Table 1 Patient Demographics

\begin{tabular}{lr}
\hline Patient characteristics $(N=28)$ & $N(\%)$ \\
\hline Sex & \\
$\quad$ Male & $18(64.2)$ \\
$\quad$ Female & $10(35.8)$ \\
Laterality & \\
$\quad$ Unilateral & $19(67.9)$ \\
$\quad$ Bilateral & $9(32.1)$ \\
Age at diagnosis (months) & \\
Mean & 17.7 \\
$\quad$ Range & $1-54$ \\
RB1 mutational status & \\
$\quad$ Not known or test not performed & $11(39.2)$ \\
Known & $17(60.7)$ \\
Germline & $6 / 17(35.3)$ \\
$\quad$ Nongermline & $10 / 17(58.8)$ \\
$\quad$ No $R B 1$ mutation identified & $1 / 17(5.9)$ \\
Secondary tumors & $2(7.1)$ \\
Distant metastasis & $4(14.2)$ \\
\hline
\end{tabular}


examination provides more thorough and conclusive determination of HRPFs; however, all cases lacking calottes were examined according to standard contemporary practices as described earlier in this paragraph.

High-risk features were determined as defined by the International Retinoblastoma Staging Working Group and included any invasion of the postlaminar optic nerve, massive choroidal invasion, anterior segment invasion, or prelaminar or laminar optic nerve invasion in conjunction with nonmassive choroidal invasion. ${ }^{21,23}$ Anaplastic grade was determined by two independent ophthalmic pathologists (C.S. and H.E.G.) blinded to patient data and included assessment of cell shape, cell wrapping, mitosis, and nuclear size, contour, and chromaticity. ${ }^{15}$ Specifically, retinocytoma was defined as cells showing unenlarged nuclei, abundant eosinophilic cytoplasm and evenly dispersed chromatin, proper differentiation as reflected by fleurettes, and the absence of mitotic figures and pleomorphism. Mild anaplasia was defined by unenlarged nuclei, differentiation (Flexner-Wintersteiner and Homer Wright rosettes), and the presence of rare mitotic figures and mild pleomorphism. Moderate anaplasia was defined by enlarged nuclei, moderate to poor differentiation, frequent mitotic figures, and moderate pleomorphism. Severe anaplasia was defined by very large hyperchromatic nuclei, poor differentiation, numerous mitotic figures, extreme pleomorphism (angular, rhomboid, or fusiform), and cell wrapping. Grade was assigned to a sample according to the highest grade identified in any focal area.

\section{Gene Expression Analysis}

Tissue cores were obtained from formalin-fixed, paraffinembedded tissue blocks for RNA preparation. Samples with

Table 2 Retinoblastoma Characteristics

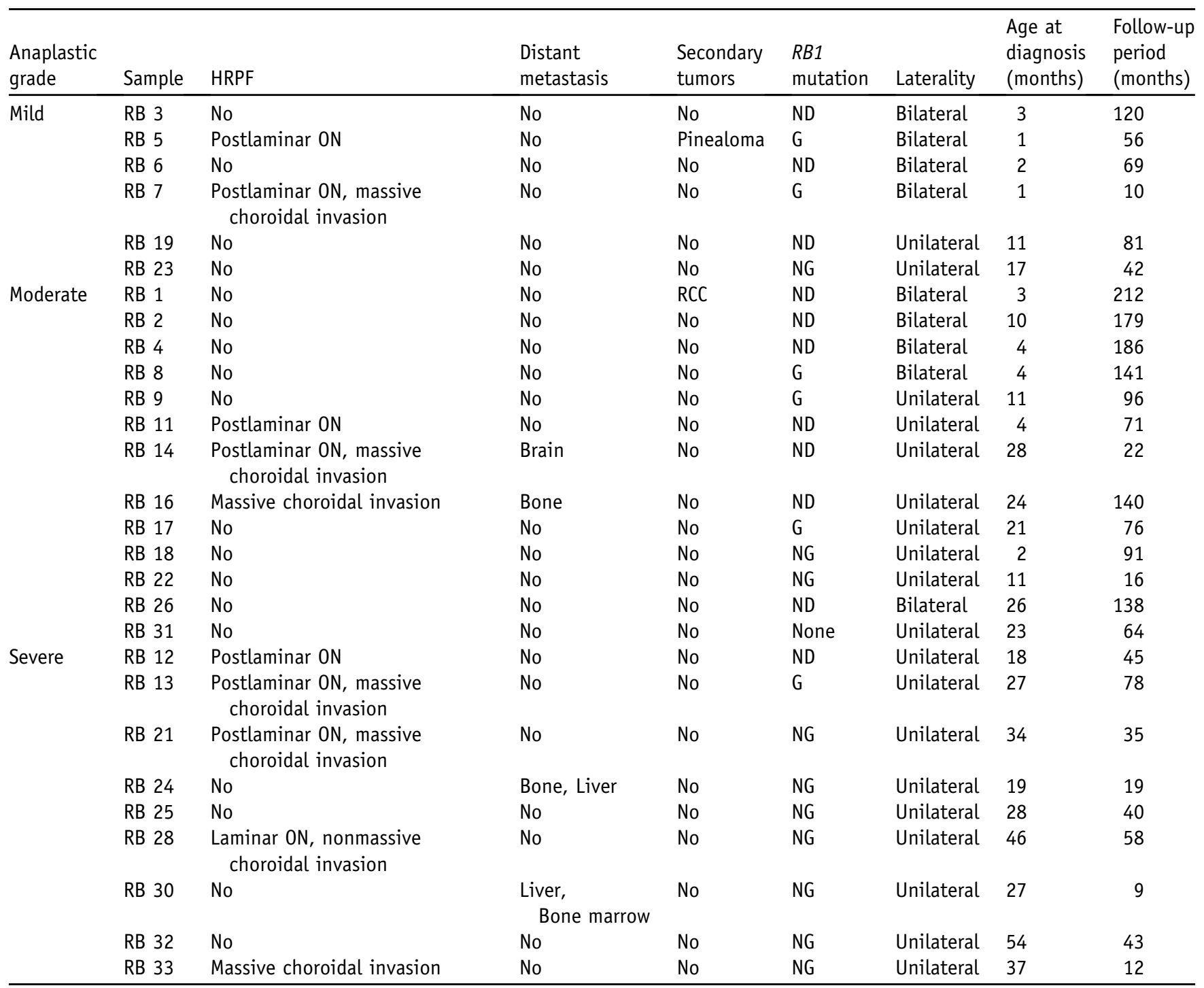

G, germline; ND, not determined; NG, nongermline; ON, optic nerve invasion; RB, retinoblastoma; RCC, renal cell carcinoma. 
less than $70 \%$ tumor content determined by histology were excluded from analysis. Total RNA was extracted using the AllPrep DNA/RNA formalin-fixed, paraffin-embedded tissue kit (Qiagen, Valencia, CA) and Mag-Bind XP formalin-fixed, paraffin-embedded tissue RNA kit (Omega Bio-tek, Norcross, GA), and RNA integrity was assessed using an Agilent 2100 Bioanalyzer (Agilent, Santa Clara, CA).

First- and second-strand cDNA synthesis, labeling, and hybridization to Affymetrix (Santa Clara, CA) Human Gene 2.0ST arrays were performed according to standard Affymetrix protocols by the Emory University Integrated Genomics Core. Data were deposited to the NCBI Gene Expression Omnibus (GEO; https://www.ncbi.nlm.nih.gov/ geo; accession number GSE110811). Samples that failed amplification (three retinoblastoma and two normal retina samples) were eliminated from the study. Microarray expression data were processed, normalized by robust multiarray average, and $\log _{2}$ transformed using the Oligo Bioconductor $\mathrm{R}$ package version 1.36.1. . $^{2,25}$

\section{Microarray Data Analysis}

Differentially expressed genes were identified using $t$-tests for each gene between groups of interest (retinoblastoma versus normal retina, severe anaplasia versus mild and moderate anaplasia, and mild and moderate anaplasia versus normal retina), and $P$ values were corrected for multiple comparisons using the Benjamini-Hochberg false discovery rate procedure. Principal component analysis (PCA) was performed on normalized expression data using the FactoMineR version $1.39^{26}$ package in R. For hierarchal clustering, affinity propagation clustering was performed with the APCluster package version 1.4.4 in $\mathrm{R}$ for all differentially expressed genes $\left(P<0.05, \mid \log _{2}\right.$ fold change $\left.\mid>0.6\right){ }^{27,28}$ Gene set enrichment analysis (GSEA) was performed with the GSEA preranked algorithm with rank determined by $-\log _{10}\left(\mathrm{p}_{\text {adj }}\right) *$ sign (fold change). ${ }^{29}$ Gene set collections searched included the hallmark, curated (C2), computational (C4), GO (C5), and oncogenic (C6) gene sets from the molecular signatures database, ${ }^{30}$ as well as gene sets generated from differentially expressed genes described by Kapatai et al. ${ }^{31}$ Linear discriminant analysis and leave-one-out cross-validation were used to identify and test genes for the prediction of anaplastic grade. ${ }^{28}$ Data were visualized using the Plotly ${ }^{32}$ and ggplot2 packages in $\mathrm{R}^{33}$

\section{Results}

\section{Patient Demographics and Retinoblastoma Histopathologic Analysis}

There were 28 retinoblastoma samples (3 with matched normal retina and 3 with retinocytoma components) that met criteria for the present study, for a total of 34 samples. The majority of patients were male $(64.2 \%)$, with age at diagnosis ranging from 1 to 54 months (Tables 1 and 2).
Nineteen retinoblastoma cases $(67.9 \%)$ presented with unilateral disease, and 6 cases developed secondary tumors (7.1\%) or distant metastases (14.2\%) (Tables 1 and 2). RBI mutational status was determined in $17(60.7 \%)$ retinoblastoma cases. Of these, six cases were found to have germline mutations. One sample had neither $R B 1$ mutations nor $M Y C N$ amplification, which drives a unique subset of unilateral retinoblastoma cases, ${ }^{34}$ but instead showed $100 \%$ hypermethylation of the $R B 1$ promoter (sample RB31). ${ }^{35,36}$ All patients with high-risk histology received adjuvant chemotherapy.

Anaplastic grade and the presence of HRPFs were determined and compared with demographic and genetic data (Table 2). Ten samples were found to have HRPFs. Nine samples (32.1\%) had severe anaplasia, 13 samples had moderate anaplasia (46.4\%), and 6 samples had mild anaplasia (21.4\%). Interestingly, HRPFs were identified in samples of each anaplastic grade from mild to severe, as described by Mendoza et $\mathrm{al}^{15}$ (Figure 1).

\section{Retinoblastoma, Retinocytoma, and Normal Retina Show Distinct Gene Expression Profiles}

To determine whether increasing degree of anaplasia in retinoblastoma is associated with different patterns of gene expression, microarray analysis was performed on the 28 retinoblastoma specimens in addition to the 3 retinocytoma and 3 normal retina specimens (Figure 2A, Supplemental Table S1). A total of 783 genes were differentially expressed in retinoblastoma compared with normal retina (583 down-regulated, 200 up-regulated) (| $\log _{2}$ fold change $>0.6 ; P<0.05)$. In retinocytoma versus normal retina, 90 genes were differentially expressed (76 downregulated, 14 up-regulated) $(P<0.05)$. Further analysis showed clear separation of samples into retinoblastoma, retinocytoma, and normal retina groups using both PCA (Figure 2B) and hierarchical clustering (Figure 2C). One

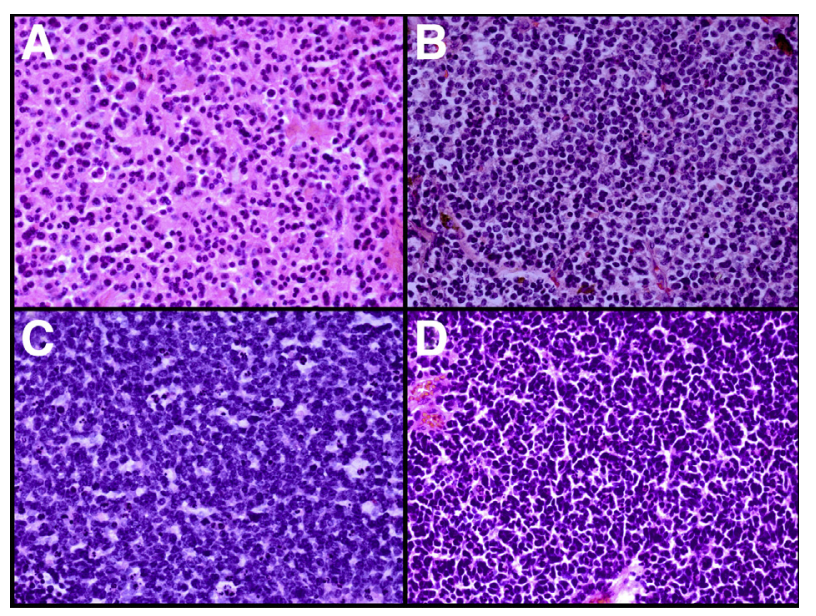

Figure 1 Anaplastic grades in retinoblastoma. A-D: Hematoxylin-eosin staining showing retinocytoma $(\mathbf{A})$ and mild $(\mathbf{B})$, moderate $(\mathbf{C})$, and severe (D) anaplasia. Original magnification, $\times 100$. 
normal retina sample (Supplemental Figure S1) did not cluster with the other normal retina samples, possibly because of inclusion of some tumor cells.

GSEA showed that genes up-regulated in retinoblastoma versus normal retina were strongly enriched for those identified previously by Kapatai et $\mathrm{al}^{31}$ as retinoblastomaassociated genes, whereas down-regulated genes were enriched for normal retina-associated genes (Supplemental Figure S2). GSEA of retinoblastoma versus normal retina using the molecular signatures database showed enrichment of gene sets related to cell-cycle checkpoint, DNA replication, and nuclear chromatin among genes up-regulated in retinoblastoma versus normal retina, whereas gene sets such as the rhodopsin pathway were enriched among downregulated genes (Figure 2, D and E, Supplemental Tables S2 and S3). This suggests that the samples not only separated into distinct groups, but also showed expected patterns of gene expression.

\section{Gene Expression Analysis Separates Retinoblastoma Samples by Anaplastic Grade But Not Presence of HRPFs}

It was next determined whether retinoblastoma with different histopathologic patterns could be distinguished by gene expression. Of interest, PCA of the 28 retinoblastoma

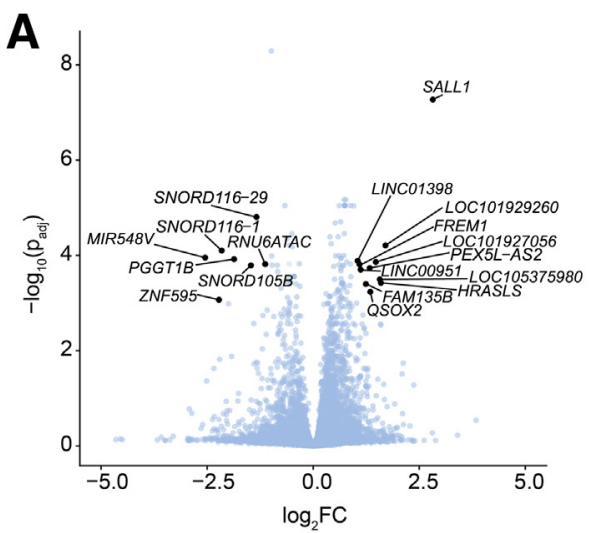

C

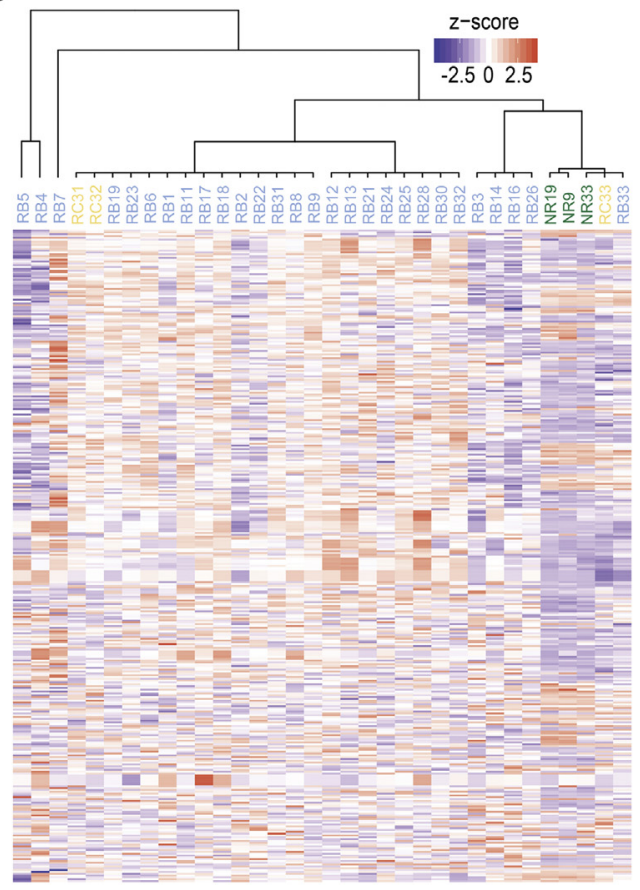

B

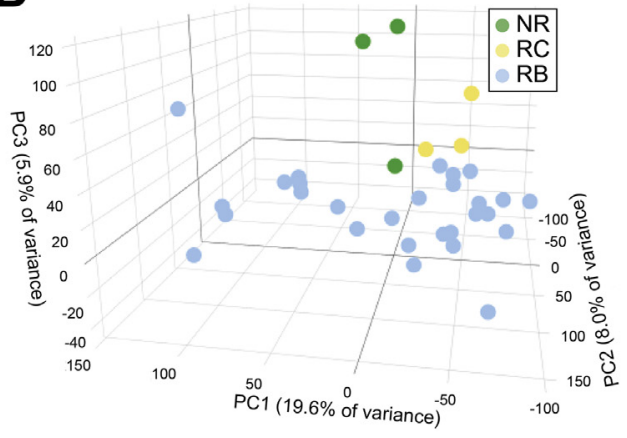

D

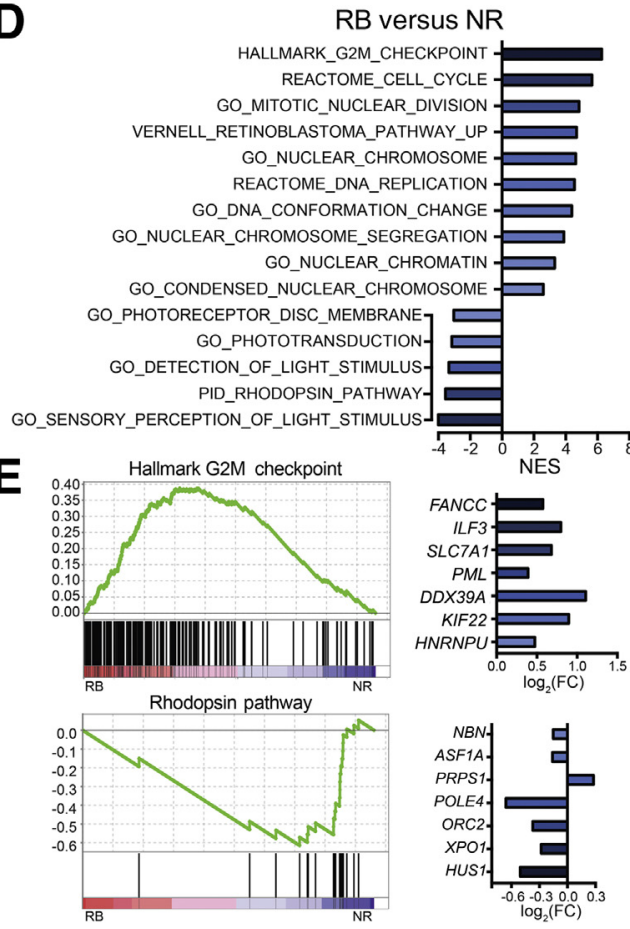

Figure 2 Gene expression profiles separate retinoblastoma, retinocytoma, and normal retina. A: Volcano plot showing differential expression of genes in retinoblastoma (RB) relative to normal retina (NR) [plot of $-\log _{10}$ of the $P$ value, adjusted for multiple comparisons ( $p_{\text {adj }}$ ), and log $\log _{2}$ of the fold change ( $F C$ )]. Genes with $\mathrm{FC}>2$ and $P<0.001$ are labeled and shown in gray. B: Principal component (PC) analysis showing sample separation into three groups: RB (blue), retinocytoma (RC; yellow), and NR (green). C: Affinity propagation clustering of all samples using genes with $\left|\log _{2} \mathrm{FC}\right|>0.6$ and $P<0.05$ in RB versus NR. D: Normalized enrichment scores (NES) of selected gene sets significantly enriched in genes up-regulated and down-regulated in RB versus NR. E: Selected enrichment plots showing gene sets enriched in genes up-regulated (hallmark G2M checkpoint) and down-regulated (rhodopsin pathway) in RB versus NR. Associated bar graphs show $\log _{2} \mathrm{FC}$ (RB versus NR) of the genes with the greatest ranked scores in their respective gene set. 
samples did not show a clear separation of tumors with versus without HRPFs (Figure 3A). In contrast, PCA of retinoblastoma samples showed grouping of tumors with severe anaplasia, suggesting a gene expression profile distinct from mild and moderate anaplastic grade tumors (Figure 3B). Interestingly, although RB33 did not group with other severe anaplasia samples (Figures 3B and Supplemental Figure S1), it showed tumor invasion of the choroid, an HRPF. RB7 similarly did not group with any samples, possibly because it possessed HRPFs but only mild anaplasia (although affinity propagation clustering shows similarity of RB7 with other mild anaplasia samples in Figure 4A). As described by Mendoza et al, ${ }^{15}$ some mild anaplasia cases can show invasion of the optic nerve and choroid whereas other tumors with severe anaplasia lack HRPFs, highlighting that HRPFs and anaplasia appear to be distinct features of retinoblastoma pathology.

The fact that mild and moderate anaplasia samples did not show clear separation from each other suggests a similar gene expression profile in these two groups. Indeed, Mendoza et al ${ }^{15}$ previously showed clinical outcomes associated with mild and moderate anaplasia to be highly similar, with $>99 \%$ and $97 \%$ 10 -year survival in cases with mild and moderate anaplasia, respectively, in contrast to $75 \%$ survival in cases with severely anaplastic tumors. Both mild and moderate anaplasia were thus considered as a single group in subsequent statistical analyses.

Hierarchical clustering using genes with $\mid \log _{2}$ fold change $\mid>0.6$ and $P<0.05$ in severe anaplasia versus mild and moderate anaplasia furthermore showed clustering according to severe versus mild-moderate anaplasia (Figure 4A). Analysis of gene expression in severe versus mild-moderate anaplasia identified several highly differentially expressed genes associated with proliferation and apoptosis of tumor cells such as CD24 (Figure 4B). ${ }^{37}$

Further analysis explored the relative number of genes dysregulated in mild-moderate anaplasia versus normal retina and severe versus mild-moderate anaplasia (Figure 4, C and D). Although the ability to detect gene expression changes may have been limited by the small number of normal retina samples and the potential for some genes to be downregulated in mild anaplasia and up-regulated in severe anaplasia (or vice versa), two clear patterns emerged. First, the majority of the highly dysregulated genes in retinoblastoma are characteristic of mild and moderate anaplasia (Figure 4, C and D). Second, few of these genes were shared among genes highly dysregulated in severe anaplasia. Together, these data suggest that unique cellular processes drive the development of severe anaplasia rather than further dysregulation of processes occurring in mild and moderate grade anaplasia.

\section{Genes Associated with Nuclear Morphology Are Differentially Expressed in Severe Anaplasia}

Given the separation of severely anaplastic from mildmoderate anaplastic grade retinoblastoma, the gene sets
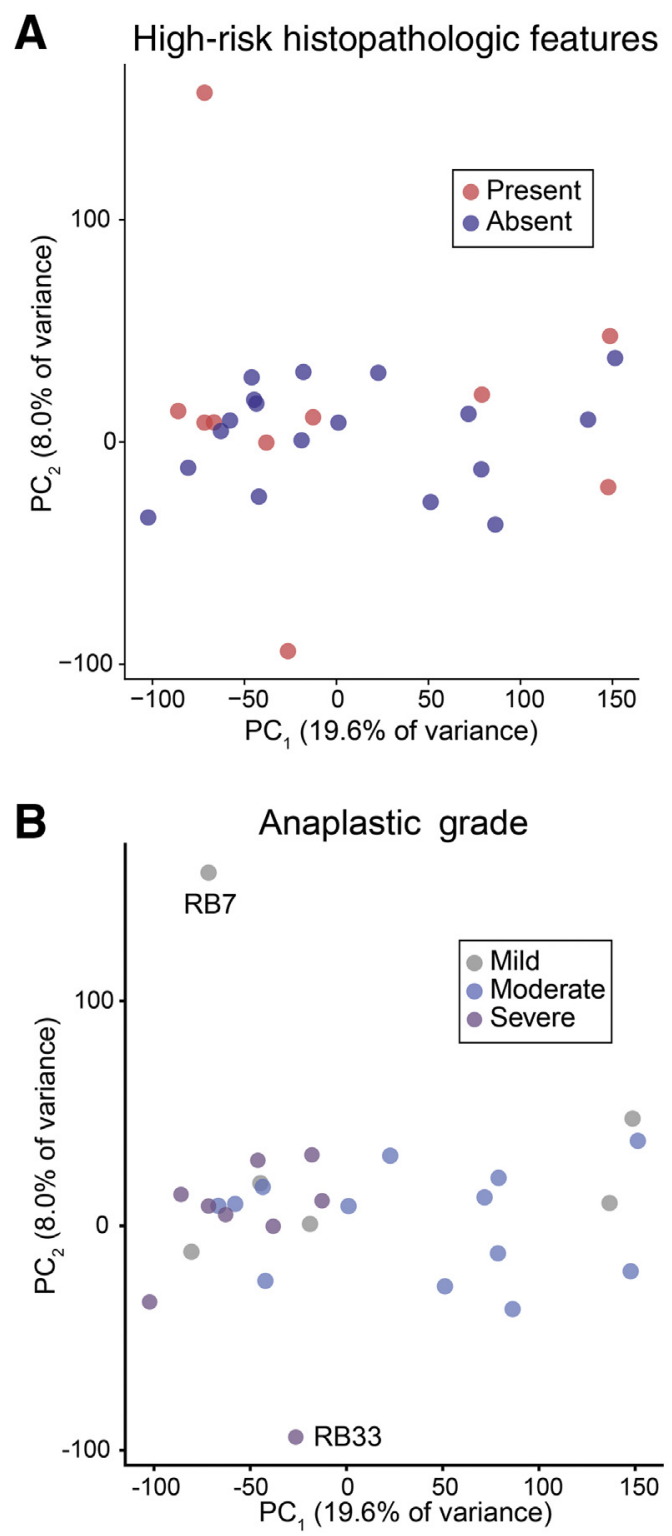

Figure 3 Gene expression can distinguish samples by anaplasia but not high-risk histopathologic features. A and B: Principal component (PC) analysis showing retinoblastoma samples by the presence of high-risk histopathologic features (A) and anaplastic grade (B).

enriched in mild-moderate anaplasia versus normal retina (Figure 4E) and in severe versus mild-moderate anaplasia (Figure 4F) were analyzed. As expected, processes related to cell cycle and proliferation were enriched among up-regulated genes in both mild-moderate anaplasia versus normal retina and severe versus mild-moderate anaplasia. Of interest, only one gene set associated with the nucleus and chromatin was significantly enriched in mild-moderate anaplasia versus normal retina, whereas several such gene sets were enriched among upregulated genes in severe versus mild-moderate anaplasia (nuclear body, chromatin modification, DNA conformation change, and so forth). Leading edge analysis of selected gene sets related to nuclear morphology showed altered 
A

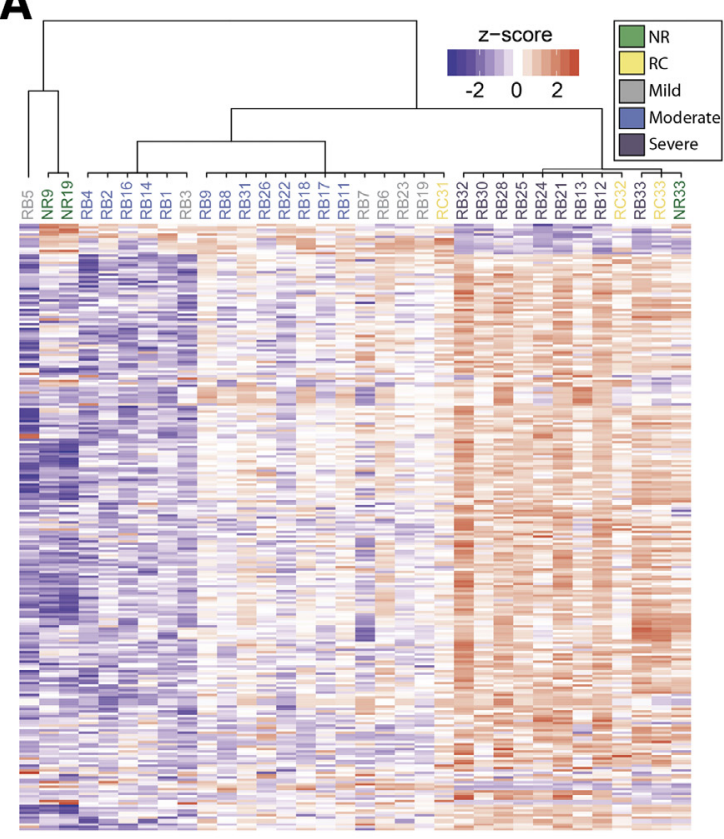

E

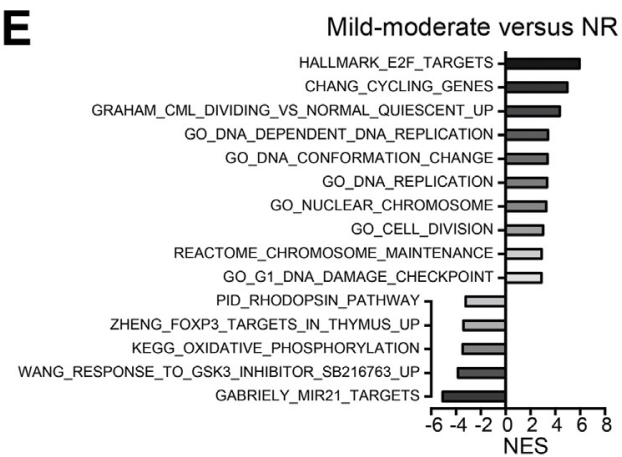

B

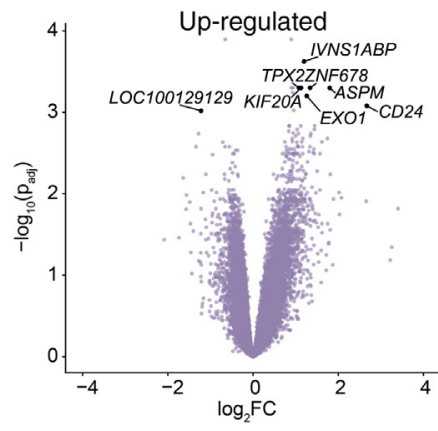

C

Highly up-regulated
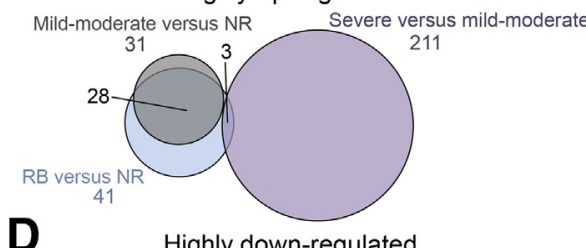

D

Highly down-regulated

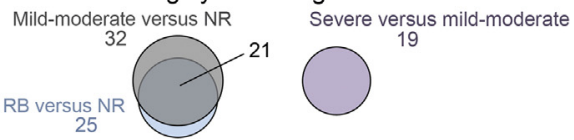

F Severe versus mild-moderate

$$
\begin{aligned}
& \text { REACTOME_CELL_CYCLE } \\
& \text { BIDUS_METASTASIS_UP - }
\end{aligned}
$$
GO MITOTIC NUCLEAR DIVISION GO_CHROMATIN MODFICATION

GO_CHROMATIN_MODIFICATION

GO_NUCLEAR_CHROMOSOME_SEGREGATON GO_NUCLEAR_BODY

GO DNA_CONFORMATION_CHANGE

GO_NUCLEAR_TRANSPORT

GO_NUCLEAR_ENVELOPE

GO_NUCLEAR_MEMBRANE-

GO_PHOTORECEPTOR_OUTLR_SEGMENT_

WORSCHECH_TUMOR_REJECTION_UP_

GO_REGULATION_OF_INFLAMMATORY_RESPONSE-

REACTOME_DEFENSINS

GO_CYTOKINE_ACTIVITY

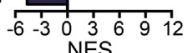

Figure 4 Retinoblastoma samples show differential patterns of gene expression by anaplastic grade. A: Hierarchical clustering using genes with $\left|\log { }_{2} \mathrm{FC}\right|>$ 0.6 and $P<0.05$ in severe anaplasia versus mild and moderate anaplasia. B: Volcano plot showing differential expression of genes in severe anaplasia relative to mild and moderate anaplasia (plot of $-\log _{10}$ of the $P$ value [adjusted for multiple comparisons $\left(p_{\text {adj }}\right)$ ] and $\log _{2} \mathrm{FC}$ ). $\mathbf{C}$ and $\mathbf{D}$ : Venn diagrams showing the number of highly up-regulated $(C)$ and highly down-regulated $(D)$ genes $\left(P<0.05,\left|\log _{2} \mathrm{FC}\right|>1\right)$ shared in all retinoblastoma (RB) versus normal retina (NR) (blue), mild-moderate anaplasia versus NR (gray), and severe versus mild-moderate anaplasia (purple). $\mathbf{E}$ and $\mathbf{F}$ : Plots of the normalized enrichment score (NES) of gene sets enriched in comparisons of mild-moderate anaplasia versus NR (E) and severe versus mild-moderate anaplasia (F).

expression of many nucleoporins and other nuclear morphology-related genes (Supplemental Table S4). In fact, expression of nucleoporins identified by leading edge analysis clearly distinguished those samples with severe anaplasia (Supplemental Figure S3A), with only one additional sample (RB6, mild anaplasia) also showing marked up-regulation of nucleoporin genes. Such enrichment of nuclear morphology-related genes among severe anaplasia samples is not unexpected given the pattern of nuclear condensation observed on histologic analysis of severe versus mild and moderate anaplasia. Indeed, severe anaplasia is defined by very large hyperchromatic nuclei and extreme pleomorphism on nuclear morphometric analysis. ${ }^{15}$ Enrichment of genes associated with nuclear shape and trafficking in severe anaplasia, then, may account for such morphologic changes observed on histology.

\section{Severe Anaplasia Shows Some Association with Previously Identified Group 2 Retinoblastoma}

Previous work by Kapatai et al ${ }^{31}$ identified two gene sets associated with retinoblastoma of differing severities and postulated cells of origin (group 1 was associated with invasive growth and possible derivation from retinal progenitor cells, and group 2 was derived from cone photoreceptors). To determine whether anaplastic grades correlated with these previously reported gene sets, ${ }^{31}$ the expression of group 1 and group 2 genes was analyzed in each retinoblastoma sample (Figure 5). Overall, a negative correlation existed between group 1-like gene expression (sample overexpression of group 1 genes and underexpression of group 2 genes relative to the mean of all samples) and group 2-like gene expression (overexpression 
of group 2 genes and underexpression of group 1 genes) $\left(r=-0.57, P=4.8 \times 10^{-4}\right)$.

GSEA analysis using the group 1 and group 2 gene lists showed significant enrichment of group 2 genes in severe versus mild-moderate anaplasia (false discovery rate-q value $=0.01$ ), but no significant associations with either group in mild-moderate anaplasia versus normal retina. Thus, although there was an overall negative correlation of group 1 and group 2 genes within our sample set, anaplastic grades did not appear to clearly segregate into either group.

\section{Photoreceptor Gene Expression Decreases in Severe Anaplasia}

Kooi et $\mathrm{al}^{38}$ recently identified a decrease in photoreceptorness that was associated with the increasing severity of retinoblastoma. In the present study, expression of cone photoreceptors indeed appeared to be somewhat decreased in mild and moderate anaplasia (Figure 6, A and B). Severely anaplastic samples showed further decreased expression of both rod and cone photoreceptors relative to normal retina and mild-moderate anaplasia. Moreover, linear discriminant analysis using the 10 photoreceptors identified by Kooi et $\mathrm{al}^{38}$ accurately predicted severe anaplasia in seven of nine cases (Figure 6C). Although the original article did not intend to analyze anaplasia in retinoblastoma, it is interesting that this small gene set is capable of distinguishing severe versus mildmoderate anaplasia and that the present study again finds photoreceptor expression to be negatively correlated with retinoblastoma severity.

\section{A Limited Gene Set Can Accurately Predict Severe Anaplasia in the Present Study}

Finally, it was determined whether genes significantly differentially expressed between severe and mild-moderate anaplasia could accurately predict anaplastic grade within our data set. In fact, four genes perfectly separated severe from mild and moderate anaplasia samples (Table 3). ${ }^{39-42}$ Linear discriminant analysis followed by leave-one-out cross-validation using the 10 most highly differentially expressed genes in severe versus mild-moderate anaplasia accurately predicted all nine cases of severe anaplasia (Figure 6C). Even using only the top two most differentially expressed genes maintained this accuracy. In contrast, prediction of mild versus moderate anaplasia was much less reliable using either 10 or 2 genes, as expected given their overall similarity in gene expression (Figures 2 and 3).

\section{Discussion}

Although novel therapies have greatly decreased both morbidity and mortality in retinoblastoma over recent decades, their full potential can be realized only through highly accurate identification of patients at risk for poor clinical outcome. ${ }^{2}$ Current means of risk stratification and determination of the need for additional therapy in retinoblastoma include assessment of clinical features, including patient age, iris neovascularization, and orbital cellulitis, ${ }^{10}$ as well as histopathologic features such as invasion of the optic nerve or choroid $^{11-13}$ and degree of cellular anaplasia. $^{15}$

Numerous oncologic studies have indicated that genebased analyses may provide more powerful and easily applicable tools to predict the need for adjuvant therapy. Gene-based tests such as that already in use for uveal melanoma facilitate rapid diagnosis and risk stratification for these diseases. ${ }^{43}$ However, such an application currently is lacking for retinoblastoma, the most common ocular tumor of childhood. ${ }^{1}$ The present study evaluated the gene expression patterns associated with a known histopathologic indicator of risk, cellular anaplasia, to determine whether
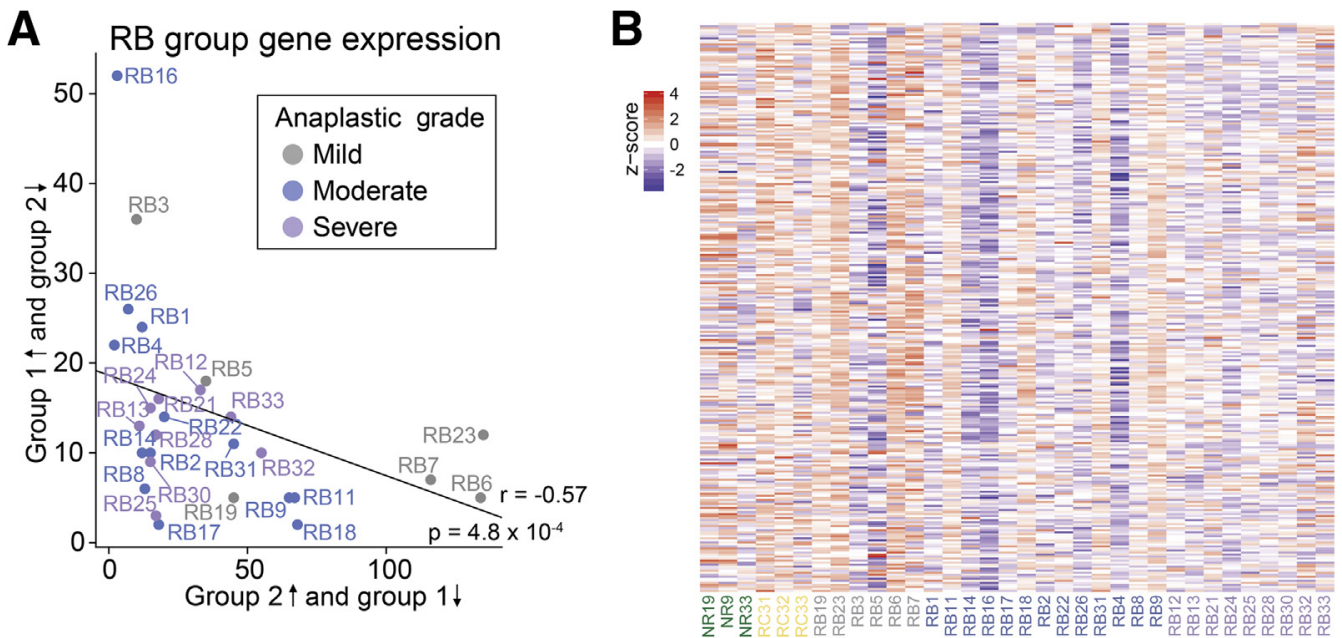

Figure 5 Anaplastic grade and group 1 and group 2 retinoblastoma. A: Plot of the number of previously reported group 1 genes ${ }^{31}$ overexpressed $(z$-score $>1)$ and group 2 genes underexpressed $(z$-score $<-1)$ (and vice versa) relative to the mean of all samples for each retinoblastoma (RB) sample. B: Heat map of group 2 gene expression in each sample. 
A
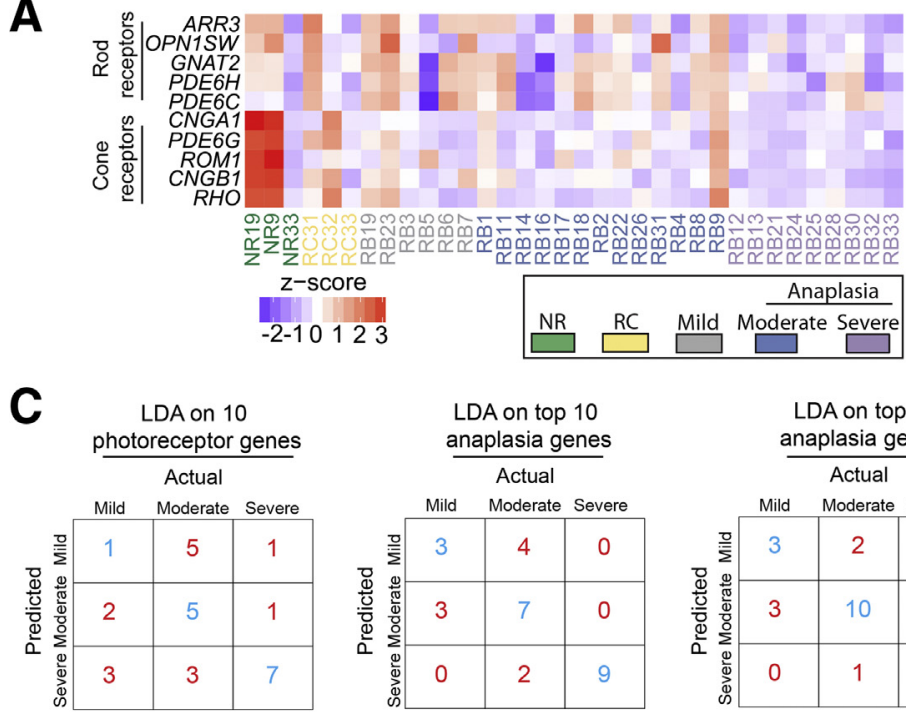
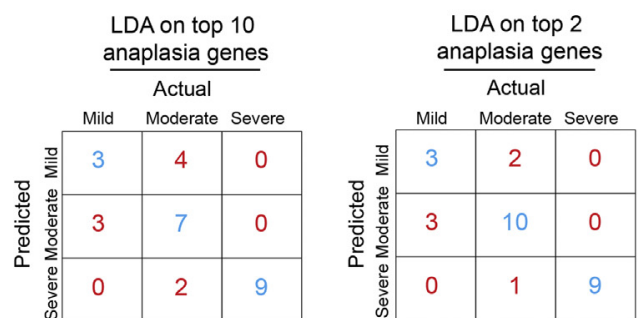

B

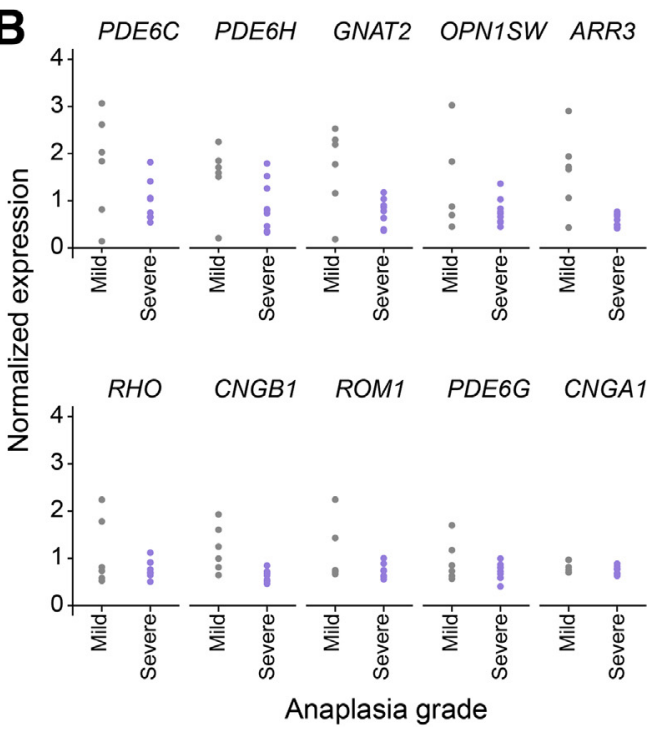

Figure 6 Photoreceptor genes are expressed differentially by anaplastic grade. A: Heat map of the expression of the previously reported 10 rod and cone photoreceptor genes within each sample. ${ }^{38}$ B: Plot of normalized expression of photoreceptor genes in samples with mild and severe anaplasia. C: Linear discriminant analysis (LDA) was used to predict anaplastic grade from the genes indicated, with results of leave-one-out cross-validation shown (top anaplasia genes selected by $P$ value ranked lowest to highest in severe versus mild-moderate anaplasia).

gene expression profiles can be used as a surrogate for histopathologic indicators of disease severity.

Results of our study indeed showed differential gene expression among retinoblastoma anaplastic grades, although interestingly there was no continuum of changes from mild to moderate to severe anaplasia. Although mild and moderate anaplasia can be distinguished from each other and severe anaplasia histopathologically, ${ }^{15}$ gene expression profiling failed to clearly separate these three grades into individual groups. Instead, mild and moderate grade anaplasia showed significant overlap, with no clear separation by either PCA or hierarchical clustering (Figures $2 \mathrm{~B}$ and $3 \mathrm{~A}$ ). Such results are not surprising given the known similarity of clinical outcomes for mild and moderate anaplasia: 10-year retinoblastoma survival rates associated with mild and moderate anaplasia are $>99 \%$ and $97 \%$, respectively, but this number significantly decreases with severe anaplasia $(75 \%) .{ }^{15}$
The disparity in gene expression between severe and mild-moderate anaplasia enabled the identification of a limited number of highly differentially expressed genes able to accurately predict all severe anaplasia samples in our data set (Figure 6C). This establishes proof of principle that particular genes could serve as surrogate markers of severe anaplasia and thus indicate high-risk retinoblastoma in a gene-based assay. However, further studies are needed to cross-validate the ability of these genes to predict severe anaplasia in additional cases of retinoblastoma.

The differences in clinical outcomes, morphology, and gene expression between severe and mild-moderate anaplasia suggest a significant shift in cellular processes between these two overarching groups. Indeed, the number of genes differentially expressed in severe compared with mild-moderate anaplasia was greater than the number of genes differentially expressed between all retinoblastoma samples versus normal retina (Figure 4, C and D).

Table 3 Four Genes Perfectly Separate Severe from Mild and Moderate Anaplasia

\begin{tabular}{|c|c|c|c|c|}
\hline Symbol & Name & $\log _{2} \mathrm{FC}$ & $p_{\text {adj }}$ & Function \\
\hline EXOC8 & Exocyst complex component 8 & 0.896602312 & 0.000127061 & $\begin{array}{l}\text { Regulates exocytosis by tethering post-Golgi vesicles } \\
\text { to the plasma membrane } \\
\text { Associated with Ras-mediated oncogenesis }{ }^{39}\end{array}$ \\
\hline CHTOP & Chromatin target of PRMT1 & 0.937928804 & 0.000500989 & $\begin{array}{l}\text { Small nuclear protein involved in } \\
\text { glioblastomagenesis }\end{array}$ \\
\hline NUCKS1 & $\begin{array}{l}\text { Nuclear casein kinase and } \\
\text { cyclin-dependent kinase } \\
\text { substrate } 1\end{array}$ & 1.126599037 & 0.051952553 & $\begin{array}{l}\text { Chromatin-associated protein involved in DNA repair } \\
\text { and homologous recombination }{ }^{41}\end{array}$ \\
\hline ADSS & Adenylosuccinate synthase & 0.931391858 & 0.000574213 & $\begin{array}{l}\text { Catalyzes the conversion of inosine monophosphate } \\
\text { to adenosine monophosphate }\end{array}$ \\
\hline
\end{tabular}

FC, fold change; $p_{a d j}, P$ value adjusted for multiple comparisons. 
Furthermore, genes dysregulated in severe anaplasia appeared to be a separate subset from those differentially expressed in mild and moderate anaplasia. Thus, it appears that a unique set of cellular processes underlies the distinction in anaplastic grades rather than up-regulation or down-regulation of processes on a continuum from mild and moderate to severe anaplasia.

Specific gene pathways underlying this gap between severe and mild-moderate anaplasia appear to be related to changes in photoreceptor expression and nuclear morphology. Indeed, all samples with severe anaplasia in our study showed down-regulation of both rod and cone photoreceptors, consistent with findings by Kooi et $\mathrm{al}^{38}$ that severe retinoblastoma is characterized by decreased photoreceptorness. Given that severe anaplasia is defined by hyperchromatic nuclei and extreme pleomorphism on nuclear morphometric analysis, ${ }^{15}$ it is of particular interest that severe anaplasia showed significant up-regulation of genes related to the regulation of nuclear morphology and chromatin condensation (Figure 4). Up-regulation of nucleoporins in particular distinguished samples with severe anaplasia (Supplemental Figure S3A). Interestingly, nucleoporin expression also was increased in a subset of poorly differentiated retinoblastoma samples from Kooi et $\mathrm{al}^{38}$ that showed the lowest photoreceptor expression (Supplemental Figure S3B). An exception to this pattern, however, appears to be the two Kooi et $\mathrm{al}^{38} R B 1^{+/+} M Y C N^{A}$ tumors, which showed low photoreceptor and nucleoporin expression. Nucleoporins with increased expression in severe anaplasia furthermore appeared to be co-regulated, with high correlation among nucleoporins in both the present data set (Supplemental Figure S3C) and in the study by Kooi et $\mathrm{al}^{38}$ (Supplemental Figure S3D). Together, these data show that increased nucleoporin expression, in combination with decreased photoreceptor expression, is characteristic of both severe anaplasia and the related, but distinct, phenotype of poor differentiation.

Nucleoporins, components of the nuclear pore complex, are known to be up-regulated in poorly differentiated cancers such as breast, ovarian, colorectal, and hematopoietic malignancies. ${ }^{44-46}$ These proteins play a central role in the dysregulation of gene expression and nuclear morphology in cancer through multiple avenues, including their ability to restrict trafficking of cell-cycle regulators and transcription factors, their roles in chromosomal tethering during the $\mathrm{G} 2$ / $M$ cell-cycle transition, and their effects on cellular motility that are hypothesized to affect metastatic potential. ${ }^{46}$ Given the up-regulation of nucleoporins in severe anaplasia in retinoblastoma in this study, it is interesting to speculate whether dysregulation of these genes contributes to both changes in nuclear morphology and the rapid and dysregulated cell cycling in more severe retinoblastoma.

In sum, this study defined the gene expression patterns of anaplastic grade in retinoblastoma. Although mild and moderate grade anaplasia are similar in terms of both gene expression and clinical outcome, severe anaplasia shows significant differences in both the number and type of dysregulated genes. Such disparity in anaplastic grades enabled the identification of specific genes able to predict severe anaplasia within the present data set, serving as a first step in determining whether particular genes could be used for clinical risk stratification. Cellular processes related to increased anaplastic severity include decreased photoreceptor expression, as previously identified, ${ }^{38}$ and markedly increased nucleoporin expression relative to mild and moderate grade anaplasia. Thus, we present here the first description of gene pathways that may underlie the increased nuclear pleomorphism and poor clinical outcomes associated with severe anaplasia in retinoblastoma. Corroboration of these findings in future retinoblastoma studies and further exploration of gene pathways underlying increased anaplastic severity will yield valuable insights into our understanding and management of this disease.

\section{Acknowledgments}

We thank Dr. J. William Harbour (Bascom Palmer Eye Institute) for helpful discussions and critical review of the manuscript, the Emory University Integrated Genomics Core for assistance in microarray data collection, and the Winship Biostatistics and Bioinformatics Shared Resource of Emory University for assistance in data analysis.

\section{Supplemental Data}

Supplemental material for this article can be found at https://doi.org/10.1016/j.ajpath.2018.06.013.

\section{References}

1. Broaddus E, Topham A, Singh AD: Incidence of retinoblastoma in the USA: 1975-2004. Br J Ophthalmol 2008, 93:21-23

2. Grossniklaus HE: Retinoblastoma. Fifty years of progress. The LXXI Edward Jackson Memorial Lecture. Am J Ophthalmol 2014, 158: 875-881.e1

3. Canturk S, Qaddoumi I, Khetan V, Ma Z, Furmanchuk A, Antoneli CB, Sultan I, Kebudi R, Sharma T, Rodriguez-Galindo C, Abramson DH, Chantada GL: Survival of retinoblastoma in lessdeveloped countries impact of socioeconomic and health-related indicators. Br J Ophthalmol 2010, 94:1432-1436

4. Shields CL, Honavar SG, Meadows AT, Shields JA, Demirci H, Singh A, Friedman DL, Naduvilath TJ: Chemoreduction plus focal therapy for retinoblastoma: factors predictive of need for treatment with external beam radiotherapy or enucleation. Am J Ophthalmol 2002, 133:657-664

5. Abramson DH, Dunkel IJ, Brodie SE, Kim JW, Gobin YP: A phase I/II study of direct intraarterial (ophthalmic artery) chemotherapy with melphalan for intraocular retinoblastoma initial results. Ophthalmology 2008, 115:1398-1404. 1404.e1

6. Shields CL, Bianciotto CG, Jabbour P, Ramasubramanian A, Lally SE, Griffin GC, Rosenwasser R, Shields JA: Intra-arterial chemotherapy for retinoblastoma: report no. 1 , control of retinal tumors, subretinal seeds, and vitreous seeds. Arch Ophthalmol 2011, 129:1399-1406 
7. Abramson DH: Super selective ophthalmic artery delivery of chemotherapy for intraocular retinoblastoma: 'chemosurgery' the first Stallard lecture. Br J Ophthalmol 2010, 94:396-399

8. Gobin YP, Dunkel IJ, Marr BP, Brodie SE, Abramson DH: Intraarterial chemotherapy for the management of retinoblastoma: four-year experience. Arch Ophthalmol 2011, 129:732-737

9. Shields CL, Mashayekhi A, Au AK, Czyz C, Leahey A, Meadows AT, Shields JA: The international classification of retinoblastoma predicts chemoreduction success. Ophthalmology 2006, 113:2276-2280

10. Kaliki S, Shields CL, Rojanaporn D, Al-Dahmash S, McLaughlin JP, Shields JA, Eagle RC: High-risk retinoblastoma based on international classification of retinoblastoma: analysis of 519 enucleated eyes. Ophthalmology 2013, 120:997-1003

11. Shields CL, Shields JA, Baez K, Cater JR, De Potter P: Optic nerve invasion of retinoblastoma. Metastatic potential and clinical risk factors. Cancer 1994, 73:692-698

12. Shields CL, Shields JA, Baez KA, Cater J, De Potter PV: Choroidal invasion of retinoblastoma: metastatic potential and clinical risk factors. Br J Ophthalmol 1993, 77:544-548

13. Haik BG, Dunleavy SA, Cooke C, Ellsworth RM, Abramson DH, Smith ME, Karcioglu ZA: Retinoblastoma with anterior chamber extension. Ophthalmology 1987, 94:367-370

14. Mabtum ED, Bonanomi MT, Lima PP, Almeida MT: Orbital retinoblastoma: case report. Arq Bras Oftalmol 2013, 76:247-249

15. Mendoza PR, Specht CS, Hubbard GB, Wells JR, Lynn MJ, Zhang Q, Kong J, Grossniklaus HE: Histopathologic grading of anaplasia in retinoblastoma. Am J Ophthalmol 2015, 159:764-776

16. Eberhart CG, Kepner JL, Goldthwaite PT, Kun LE, Duffner PK, Friedman HS, Strother DR, Burger PC: Histopathologic grading of medulloblastomas. Cancer 2002, 94:552-560

17. Jijelava KP, Grossniklaus HE: Diffuse anterior retinoblastoma: a review. Saudi J Ophthalmol 2013, 27:135-139

18. Shields CL, Ghassemi F, Tuncer S, Thangappan A, Shields JA: Clinical spectrum of diffuse infiltrating retinoblastoma in 34 consecutive eyes. Ophthalmology 2008, 115:2253-2258

19. Reese A: Tumors of the Eye. ed 3. Hagerstown, MD, Harper and Row, 1976

20. Murphree AL: Intraocular retinoblastoma: the case for a new group classification. Ophthalmol Clin North Am 2005, 18:41-53

21. Sastre X, Chantada GL, Doz F, Wilson MW, De Davila MTG, Rodríguez-Galindo C, Chintagumpala M, Chévez-Barrios P: Proceedings of the Consensus Meetings from the International Retinoblastoma Staging Working Group on the pathology guidelines for the examination of enucleated eyes and evaluation of prognostic risk factors in retinoblastoma. Arch Pathol Lab Med 2009, 133:1199-1202

22. Edge SB, Compton CC: The American Joint Committee on Cancer: the 7th edition of the AJCC cancer staging manual and the future of TNM. Ann Surg Oncol 2010, 17:1471-1474

23. Eagle RC Jr: High-risk features and tumor differentiation in retinoblastoma: a retrospective histopathologic study. Arch Pathol Lab Med 2009, 133:1203-1209

24. Carvalho BS, Irizarry RA: A framework for oligonucleotide microarray preprocessing. Bioinformatics 2010, 26:2363-2367

25. Gentleman RC, Carey VJ, Bates DM, Bolstad B, Dettling M, Dudoit S, Ellis B, Gautier L, Ge Y, Gentry J, Hornik K, Hothorn T, Huber W, Iacus S, Irizarry $\mathrm{R}$, Leisch $\mathrm{F}$, Li C, Maechler M, Rossini AJ, Sawitzki G, Smith C, Smyth G, Tierney L, Yang JYH, Zhang J: Bioconductor: open software development for computational biology and bioinformatics. Genome Biol 2004, 5:R80

26. Lê S, Josse J, Husson F: FactoMineR: an R package for multivariate analysis. J Stat Softw 2008, 25:1-18

27. Bodenhofer U, Kothmeier A, Hochreiter S: APCluster: an R package for affinity propagation clustering. Bioinformatics 2011, 27:2463-2464

28. Frey BJ, Dueck D: Clustering by passing messages between data points. Science 2007, 315:972-976
29. Subramanian A, Tamayo P, Mootha VK, Mukherjee S, Ebert BL, Gillette MA, Paulovich A, Pomeroy SL, Golub TR, Lander ES, Mesirov JP: Gene set enrichment analysis: a knowledge-based approach for interpreting genome-wide expression profiles. Proc Natl Acad Sci U S A 2005, 102:15545-15550

30. Liberzon A, Subramanian A, Pinchback R, Thorvaldsdottir H, Tamayo P, Mesirov JP: Molecular signatures database (MSigDB) 3.0. Bioinformatics 2011, 27:1739-1740

31. Kapatai G, Brundler MA, Jenkinson H, Kearns P, Parulekar M, Peet AC, McConville CM: Gene expression profiling identifies different sub-types of retinoblastoma. Br J Cancer 2013, 109:512-525

32. Plotly Technologies Inc.: Collaborative Data Science. Montreal, QC, Plotly Technologies Inc., 2015

33. Wickham H: ggplot2: Elegant Graphics for Data Analysis, ed 2. New York, NY: Springer-Verlag, 2016

34. Rushlow DE, Mol BM, Kennett JY, Yee S, Pajovic S, Thériault BL, Prigoda-Lee NL, Spencer C, Dimaras H, Corson TW, Pang R, Massey C, Godbout R, Jiang Z, Zacksenhaus E, Paton K, Moll AC, Houdayer C, Raizis A, Halliday W, Lam WL, Boutros PC, Lohmann D, Dorsman JC, Gallie BL: Characterisation of retinoblastomas without RB1 mutations: genomic, gene expression, and clinical studies. Lancet Oncol 2013, 14:327-334

35. Ohtani-Fujita N, Fujita T, Aoike A, Osifchin NE, Robbins PD, Sakai T: CpG methylation inactivates the promoter activity of the human retinoblastoma tumor-suppressor gene. Oncogene 1993, 8: 1063-1067

36. Stirzaker C, Millar DS, Paul CL, Warnecke PM, Harrison J, Vincent PC, Frommer M, Clark SJ: Extensive DNA methylation spanning the Rb promoter in retinoblastoma tumors. Cancer Res 1997, 57:2229-2237

37. Li J, Li C, Yuan H, Gong F: Clinical value of CD24 expression in retinoblastoma. J Biomed Biotechnol 2012, 2012:1-6

38. Kooi IE, Mol BM, Moll AC, van der Valk P, de Jong MC, de Graaf P, van Mil SE, Schouten-van Meeteren AYN, Meijers-Heijboer H, Kaspers GL, te Riele H, Cloos J, Dorsman JC: Loss of photoreceptorness and gain of genomic alterations in retinoblastoma reveal tumor progression. EBioMedicine 2015, 2:660-670

39. Issaq SH, Lim KH, Counter CM: Sec5 and Exo84 foster oncogenic Ras-mediated tumorigenesis. Mol Cancer Res 2010, 8 : 223-231

40. Takai H, Masuda K, Sato T, Sakaguchi Y, Suzuki T, Suzuki T, KoyamaNasu R, Nasu-Nishimura Y, Katou Y, Ogawa H, Morishita Y, KozukaHata H, Oyama M, Todo T, Ino Y, Mukasa A, Saito N, Toyoshima C, Shirahige K, Akiyama T: 5-hydroxymethylcytosine plays a critical role in glioblastomagenesis by recruiting the CHTOP-methylosome complex. Cell Rep 2014, 9:48-60

41. Parplys AC, Zhao W, Sharma N, Groesser T, Liang F, Maranon DG, Leung SG, Grundt K, Dray E, Idate R, Østvold AC, Schild D, Sung P, Wiese C: NUCKS1 is a novel RAD51AP1 paralog important for homologous recombination and genome stability. Nucleic Acids Res 2015, 43:9817-9834

42. Stayton MM, Rudolph FB, Fromm HJ: Regulation, genetics, and properties of adenylosuccinate synthetase: a review. Current Topics in Cellular Regulation, vol. 22. Edited by Horecker BL, Stadman ER. Cambridge, MA: Academic Press, 1983. pp. 103-141

43. Harbour JW: A prognostic test to predict the risk of metastasis in uveal melanoma based on a 15-gene expression profile. Methods Mol Biol 2014, 1102:427-440

44. Kabachinski G, Schwartz TU: The nuclear pore complex-structure and function at a glance. J Cell Sci 2015, 128:423-429

45. Strambio-De-Castillia C, Niepel M, Rout MP: The nuclear pore complex: bridging nuclear transport and gene regulation. Nat Rev Mol Cell Biol 2010, 11:490-501

46. Chow K-H, Factor RE, Ullman KS: The nuclear envelope environment and its cancer connections. Nat Rev Cancer 2012, 12:196-209 